\title{
NOTIONS OF SPANNING SURFACE EQUIVALENCE
}

\author{
JULIAN R. EISNER
}

Abstract. We show that two natural notions of spanning surface equivalence differ for minimal spanning surfaces of knots in $S^{3}$.

1. Introduction. Every (tame) knot in $S^{3}$ is spanned by a (tame) orientable surface [8]; an orientable spanning surface of smallest possible genus is called a minimal spanning surface. Spanning surfaces $F$ and $F^{\prime}$ of a knot $K$ in $S^{3}$ are weakly equivalent if there is an autohomeomorphism of $S^{3}$ taking $F$ to $F^{\prime}$ and preserving the orientations of both $S^{3}$ and $K$. The surfaces $F$ and $F^{\prime}$ are strongly equivalent if there is an isotopic deformation of $S^{3}$, fixing $K$ (throughout the isotopy), and taking $F$ to $F^{\prime}$. Alford, Daigle, Lyon, Schaufele, and Trotter have given examples of knots which have minimal spanning surfaces $F$ and $F^{\prime}$ which are not weakly equivalent [1]-[4], [6], [10]. We show here that there are knots $K$ possessing minimal spanning surfaces $F$ and $F$ which are weakly equivalent but not strongly equivalent.

2. The construction. Write $S^{3}=R^{3} \cup \infty$, and set

$$
\begin{aligned}
B_{1} & =\left\{\left(y_{1}, y_{2}, y_{3}\right): y_{1} \geqslant 0\right\} \cup \infty, \quad B_{2}=\left\{\left(y_{1}, y_{2}, y_{3}\right): y_{1} \leqslant 0\right\} \cup \infty, \\
S^{2} & =\left\{\left(y_{1}, y_{2}, y_{3}\right): y_{1}=0\right\} \cup \infty, \\
a & =\left\{\left(y_{1}, y_{2}, y_{3}\right): y_{1}=0,-1 \leqslant y_{2} \leqslant 1, y_{3}=0\right\}
\end{aligned}
$$

and $x=(0,0,1)$. Let $h$ be the orientation preserving autohomeomorphism of $S^{3}$ given by $h\left(y_{1}, y_{2}, y_{3}\right)=\left(-y_{1},-y_{2}, y_{3}\right)$; note that $h(a)=a$ and that $h$ reverses the orientation of $a$. By [1], [2], we can find a knot $k$ possessing minimal spanning surfaces $S$ and $S^{\prime}$ with $\pi_{1}\left(S^{3}-S\right) \neq \pi_{1}\left(S^{3}-S^{\prime}\right)$. We may assume that $k, S$, and $S^{\prime}$ lie in $B_{1}$ and that $k \cap S^{2}=S \cap S^{2}=S^{\prime} \cap S^{2}$ $=a$. Let $K$ be the composite $\operatorname{knot} k \# h(k)=(k \cup h(k))-$ int $(a)$, and set $F=S \cup h\left(S^{\prime}\right)$ and $F^{\prime}=S^{\prime} \cup h(S) ; F$ and $F^{\prime}$ are minimal spanning surfaces of $K\left[5\right.$, p. 141]. Since $h^{2}=$ id, $h(F)=h\left(S \cup h\left(S^{\prime}\right)\right)=h(S) \cup S^{\prime}=F^{\prime}$. Also, since $h$ reverses the orientation of $a, h$ preserves the orientation of $K$. Therefore, $F$ and $F^{\prime}$ are weakly equivalent. We will show, however, that $F$ and $F^{\prime}$ are not strongly equivalent.

3. Distinguishing between $F$ and $F^{\prime}$. We will prove that $F$ and $F^{\prime}$ are not strongly equivalent by demonstrating that if they were, it would then follow

Received by the editors January 10, 1975.

AMS (MOS) subject classifications (1970). Primary 55A25; Secondary 55A05, 55A35.

Key words and phrases. Knot, composite knot, minimal spanning surface, isotopic deformation.

(1) American Mathematical Society 1976 
that $\pi_{1}\left(S^{3}-S, x\right) \simeq \pi_{1}\left(S^{3}-S^{\prime}, x\right)$, which is, by hypothesis, false. The fact that $\pi_{1}\left(S^{3}-S, x\right)$ would be isomorphic to $\pi_{1}\left(S^{3}-S^{\prime}, x\right)$ if $F$ and $F^{\prime}$ were strongly equivalent will follow from a careful examination of the spaces and groups involved in our construction, together with an application of the algebraic consequences of the existence of a strong equivalence.

Let $u_{1}, u_{2}, o_{1}$, and $o_{2}$ be inclusion maps in the following commutative diagram:

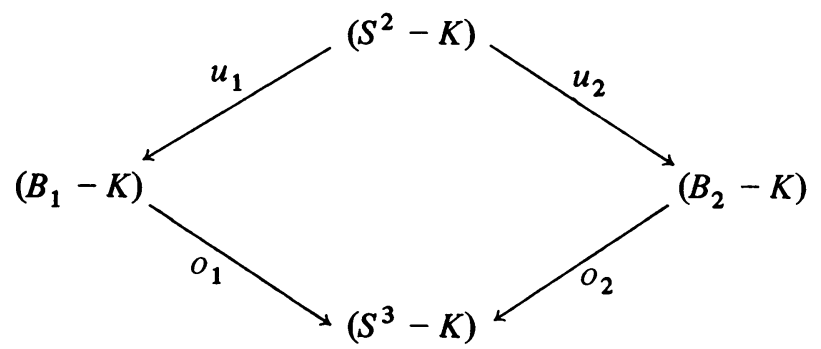

(We let $(A-B)$ denote the set of all points which are in $A$ but not in $B$, even when $B$ is not a subset of $A$. Thus, for example, $\left(S^{2}-K\right)=\left(S^{2}\right.$ minus two points).) Letting $n$ and $n_{2}$ be the natural maps in the commutative diagram

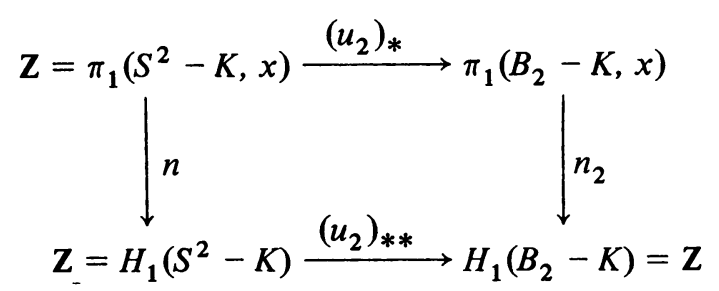

and observing that $n$ and $\left(u_{2}\right)_{* *}$ are isomorphisms, we see that the homomorphism $\left(u_{2}\right)_{*}$ has a left inverse $v=n^{-1} \circ\left(\left(u_{2}\right)_{* *}\right)^{-1} \circ n_{2}$, which abelianizes $\pi_{1}\left(B_{2}-K, x\right)$. Letting $\phi_{1}$ be the identity homomorphism of $\pi_{1}\left(B_{1}-K, x\right)$ to itself and letting $\phi_{2}: \pi_{1}\left(B_{2}-K, x\right) \rightarrow \pi_{1}\left(B_{1}-K, x\right)$ be the homomorphism $\left(u_{1}\right)_{*} \circ v$, we see that

$$
\phi_{1} \circ\left(u_{1}\right)_{*}=\left(u_{1}\right)_{*}=\left(u_{1}\right)_{*} \circ v \circ\left(u_{2}\right)_{*}=\phi_{2} \circ\left(u_{2}\right)_{*},
$$

so that, by the Seifert-Van Kampen theorem, there is a homomorphism $\phi: \pi_{1}\left(S^{3}-K, x\right) \rightarrow \pi_{1}\left(B_{1}-K, x\right)$ such that $\phi \circ\left(o_{1}\right)_{*}=\phi_{1}=$ id and $\phi$ $\circ\left(o_{2}\right)_{*}=\phi_{2}=\left(u_{1}\right)_{*} \circ v$. Since $v$ abelianizes $\pi_{1}\left(B_{2}-K, x\right)$, we see that $\phi$ $\circ\left(\mathrm{O}_{2}\right)_{*}$ kills the commutator subgroup of $\pi_{1}\left(B_{2}-K, x\right)$.

Now consider the following commutative diagram, in which all arrows are given by inclusion:

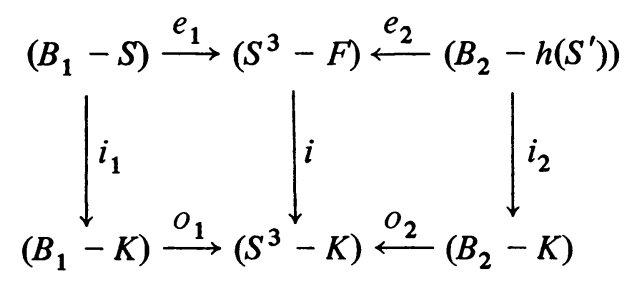


By the Seifert-Van Kampen theorem, $\phi \circ i_{*}\left(\pi_{1}\left(S^{3}-F, x\right)\right)$ is generated by $\phi \circ i_{*} \circ\left(e_{1}\right)_{*}\left(\pi_{1}\left(B_{1}-S, x\right)\right)$ and $\phi \circ i_{*} \circ\left(e_{2}\right)_{*}\left(\pi_{1}\left(B_{2}-h\left(S^{\prime}\right), x\right)\right)$. Now

$$
\phi \circ i_{*} \circ\left(e_{1}\right)_{*}=\phi \circ\left(o_{1}\right)_{*} \circ\left(i_{1}\right)_{*}=\mathrm{id} \circ\left(i_{1}\right)_{*}=\left(i_{1}\right)^{*} \text {, }
$$

which, by Dehn's lemma and the loop theorem, is injective since $S$ is minimal (cf. [7, pp. 27-32] or [9]). Consequently

$$
\phi \circ i_{*} \circ\left(e_{1}\right)_{*}\left(\pi_{1}\left(B_{1}-S, x\right)\right) \simeq \pi_{1}\left(B_{1}-S, x\right) \simeq \pi_{1}\left(S^{3}-S, x\right) .
$$

Also, $\phi \circ i_{*} \circ\left(e_{2}\right)_{*}=\phi \circ\left(o_{2}\right)_{*} \circ\left(i_{2}\right)_{*}=0$, since the image of $\left(i_{2}\right)_{*}$ is in the commutator subgroup of $\pi_{1}\left(B_{2}-K, x\right)$, which is killed by $\phi \circ\left(o_{2}\right)_{*}$. Therefore, $\phi \circ i_{*}\left(\pi_{1}\left(S^{3}-F, x\right)\right) \simeq \pi_{1}\left(S^{3}-S, x\right)$. Similarly, letting $i^{\prime}:\left(S^{3}-F^{\prime}\right)$ $\rightarrow\left(S^{3}-K\right)$ be the inclusion map, we have

$$
\phi \circ\left(i^{\prime}\right)_{*}\left(\pi_{1}\left(S^{3}-F^{\prime}, x\right)\right) \simeq \pi_{1}\left(S^{3}-S^{\prime}, x\right) .
$$

THEOREM. $F$ and $F^{\prime}$ are not strongly equivalent.

Proof. Suppose the contrary. Then there is an isotopic deformation $J: S^{3} \times I \rightarrow S^{3}$ such that, for each $t, J_{t}(K)=K$, and $J_{1}(F)=F^{\prime}$. Since $x \in\left(S^{3}-\left(F \cup F^{\prime}\right)\right)$, we may assume as well that $J_{1}(x)$, $=x$. Then $\left(J_{1} \mid S^{3}-K\right)_{*}$ is the inner automorphism of $\pi_{1}\left(S^{3}-K, x\right)$ given by conjugation by the element of $\pi_{1}\left(S^{3}-K, x\right)$ represented by the path of $x$ during the isotopy $J$. We see also that

$$
\left(J_{1} \mid S^{3}-K\right)_{*}\left(u_{*}\left(\pi_{1}\left(S^{3}-F, x\right)\right)\right)=\left(i^{\prime}\right)_{*}\left(\pi_{1}\left(S^{3}-F^{\prime}, x\right)\right),
$$

since

$$
J_{1}\left(S^{3}-F\right)=\left(S^{3}-F^{\prime}\right) .
$$

Therefore, $i_{*}\left(\pi_{1}\left(S^{3}-F, x\right)\right)$ and $\left(i^{\prime}\right)_{*}\left(\pi_{1}\left(S^{3}-F^{\prime}, x\right)\right)$ are conjugate subgroups of $\pi_{1}\left(S^{3}-K, x\right)$. Consequently

$$
\phi \circ i_{*}\left(\pi_{1}\left(S^{3}-F, x\right)\right) \quad \text { and } \phi \circ\left(i^{\prime}\right)_{*}\left(\pi_{1}\left(S^{3}-F^{\prime}, x\right)\right)
$$

are conjugate subgroups of $\pi_{1}\left(B_{1}-K, x\right)$; in particular,

$$
\phi \circ i_{*}\left(\pi_{1}\left(S^{3}-F, x\right)\right) \simeq \phi \circ\left(i^{\prime}\right)_{*}\left(\pi_{1}\left(S^{3}-F^{\prime}, x\right)\right),
$$

or $\pi_{1}\left(S^{3}-S, x\right) \simeq \pi_{1}\left(S^{3}-S^{\prime}, x\right)$, a contradiction. $\|$

\section{BIBLIOGRAPHY}

1. W. R. Alford, Complements of minimal spanning surfaces of knots are not unique, Ann. of Math. (2) 91 (1970), 419-424. MR 40 \#6527.

2. W. R. Alford and C. B. Schaufele, Complements of minimal spanning surfaces of knots are not unique. II, Topology of Manifolds (Proc. Univ. of Georgia, Athens, Ga., 1969), Markham, Chicago, Ill., 1970, pp. 87-96. MR 44 \#5947.

3. R. J. Daigle, Complements of minimal surfaces (preprint).

4. - More on complements of minimal spanning surfaces, Rocky Mountain J. Math. 3 (1973), 473-482.

5. R. H. Fox, A quick trip through knot theory, Topology of 3-Manifolds and Related Topics 
(Proc. Univ. of Georgia Inst., 1961), Prentice-Hall, Englewood Cliffs, N. J., 1962, pp. 120-167. MR 25 \#3522.

6. H. C. Lyon, Simple knots without unique minimal surfaces, Proc. Amer. Math. Soc. 43 (1974), 449-454.

7. L. P. Neuwirth, Knot groups, Ann. of Math. Studies, no. 56, Princeton Univ. Press, Princeton, N. J., 1965. MR 31 \# 734.

8. H. Seifert, Uber das Geschlecht von Knoten, Math. Ann. 110 (1934), 571-592.

9. J. R. Stallings, On fibering certain 3-manifolds, Topology of 3-Manifolds and Related Topics (Proc. Univ. of Georgia Inst., 1961, Prentice-Hall, Englewood Cliffs, N. J., 1962, pp. 95-100. MR 28 \# 1600.

10. H. F. Trotter, Some knots spanned by more than one unknotted surface of minimal genus, Ann. of Math. Studies, no. 84, Princeton Univ. Press, Princeton, N. J., 1975, pp. 51-62.

Department of Mathematics, Princeton University, Princeton, New Jersey 08540

Current address: Department of Mathematics, Columbia University, New York, New York 10027 